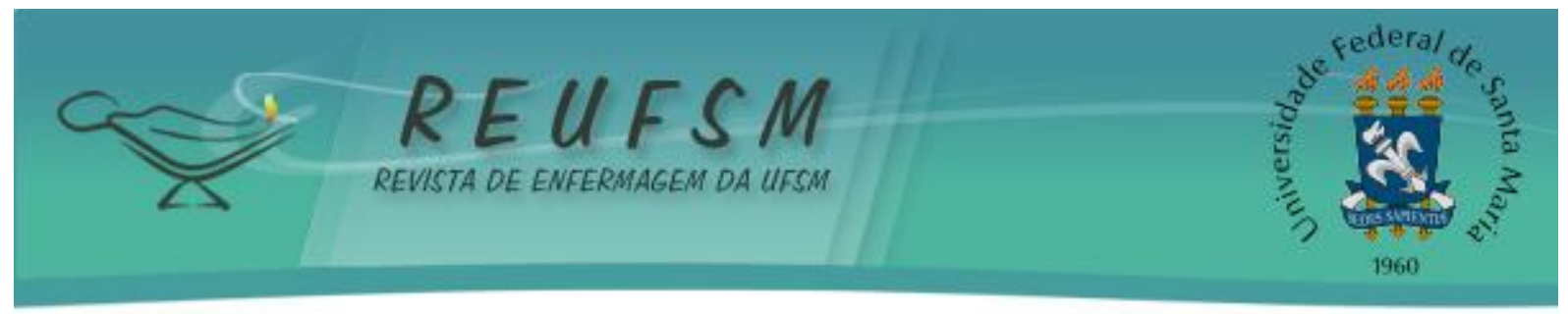

ARTIGO ORIGINAL

\title{
CONTRIBUIÇÕES DE ENCONTROS DE SENSIBILIZAÇÃO E CUIDADO PARA ACADÊMICOS DE ENFERMAGEM
}

CONTRIBUTIONS OF MEETINGS OF AWARENESS AND CARE FOR NURSING STUDENTS CONTRIBUCIONES DE LOS ENCUENTROS DE SENSIBILIZACIÓN Y CUIDADO PARA LOS ESTUDIANTES DE
ENFERMERIAA

Luciara Fabiane Sebold ${ }^{1}$

Silvana Silveira Kempfer ${ }^{2}$

Telma Elisa Carraro ${ }^{3}$

Vera Radünz ${ }^{4}$

RESUMO: Objetivo: relatar as contribuições que os encontros de sensibilização e cuidado proporcionaram aos acadêmicos de enfermagem de uma universidade pública do Sul do Brasil. Método: pesquisa qualitativa de abordagem Convergente Assistencial (PCA), desenvolvida com 42 acadêmicos de enfermagem, em uma universidade pública da Região Sul do Brasil. Coleta dos dados deu-se durante quatro encontros. A análise dos dados seguiu PCA. Resultados: os acadêmicos puderam experimentar um espaço descontraído, com atividades lúdicas, as quais proporcionaram a expressão de práticas cotidianas, sensibilizando para cuidar de si e o cuidado com o outro. Conclusões: refletir sobre a realidade, de forma crítica criativa, é a melhor maneira de buscar a expressão do potencial para além da reflexão e a transformação de alguns conceitos do dia a dia do enfermeiro.

Descritores: Estudantes de enfermagem; Enfermagem; Prática de grupo; Cuidados de enfermagem; Promoção da saúde.

ABSTRACT: Objective: to report the contributions awareness meetings and care provided to nursing students at a public university in southern Brazil. Method: qualitative Research for Convergent Care approach (PCA), developed with 42 nursing students in a public university in Southern Brazil. Data collection occurred during four meetings. Data analysis followed PCA. Results: the students were able to experience a relaxed space with recreational activities which have brought the expression of everyday practices, raise awareness of caring for themselves and care for others. Conclusions: reflect on the reality, critically creative, is the best way to get the expression of the potential beyond the reflection and transformation of some concepts of everyday nursing.

Descriptors: Students of nursing; Nursing; Group practice; Nursing care; Health promotion.

RESUMEM: Objetivo: reportar las contribuciones que las reuniones de sensibilización y atención proporcionaran a los estudiantes de enfermería en una universidad pública en el Sur de Brasil. Método: investigación cualitativa para la aproximación Convergente Asistencial (PCA), desarrollado con 42 estudiantes de enfermería en una universidad pública en el Sur de Brasil. Los datos fueron recolectados en cuatro reuniones. El análisis de datos seguido de PCA.

\footnotetext{
${ }^{1}$ Enfermeira. Doutora em Enfermagem pelo Programa de Pós-Graduação em Enfermagem pela Universidade Federal de Santa Catarina. Bolsista PNPD. Email: fabisebold@gmail.com

${ }^{2}$ Enfermeira. Doutoranda pelo Programa de Pós-Graduação em Enfermagem da Universidade Federal de Santa Catarina. Bolsista CNPQ. Email: silvanakempfer@yahoo.com.br

${ }^{3}$ Enfermeira. Doutora em Enfermagem pelo Programa de Pós-Graduação em Enfermagem pela Universidade Federal de Santa Catarina. Professora do Departamento de Enfermagem UFSC. Email: telmacarraro@ccs.ufsc ${ }^{4}$ Enfermeira. Doutora em Enfermagem pelo Programa de Pós-Graduação em Enfermagem pela Universidade Federal de Santa Catarina. Professora do Departamento de Enfermagem UFSC. Email: veraradunz@ccs.ufsc
} 


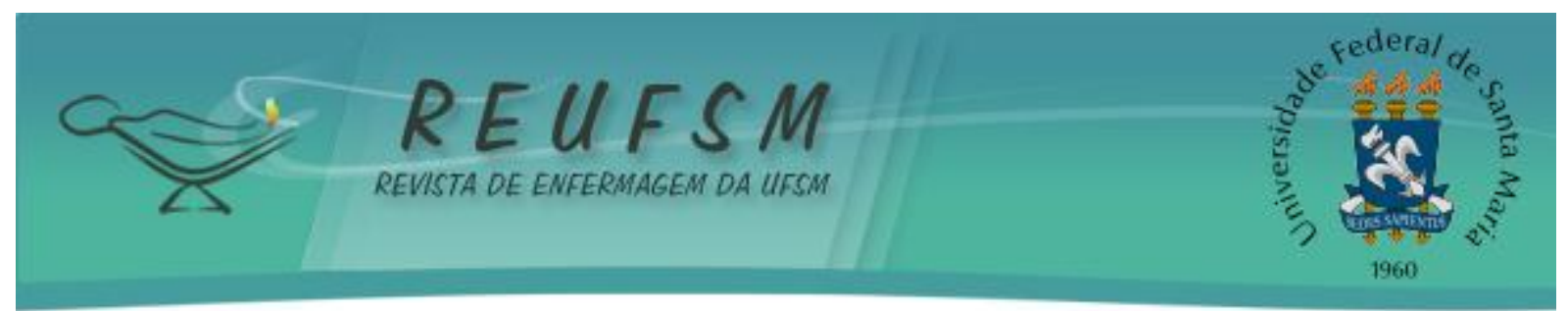

Resultados: los estudiantes pudieron experimentar un espacio relajado, con actividades recreativas que han llevado a la expresión de las prácticas cotidianas, sensibilizar sobre el cuidado de sí mismos y cuidar de los demás. Conclusiones: reflexionar sobre la realidad, la crítica creativa, es la mejor manera de obtener la expresión del potencial más allá de la reflexión y la transformación de algunos conceptos de la rutina del enfermero.

Descriptores: Estudiantes de enfermería; Enfermería; Práctica de grupo; Atención de enfermería; Promoción de la salud.

\section{INTRODUÇÃO}

O processo de ensino e aprendizagem apresenta nuances que merecem ser reconhecidas como imprescindíveis para o verdadeiro aprendizado, sendo assim, precisa ter sentido para os sujeitos em seu cotidiano. Este processo torna-se significativo à medida que as estruturas do conhecimento sejam relacionadas à temática a qual se quer aprender. Uma das estratégias para que aconteça o aprendizado é sensibilizar os alunos para a busca do conhecimento e, que este tenha um papel importante em suas vidas.

Neste aspecto, a sensibilização para a importância do cuidar de si e a evitabilidade do sobrepeso representa um reconhecimento do complexo sistema de fatores inter-relacionados, os quais os acadêmicos estão inseridos em suas realidades. 0 cuidar de si, neste momento, expressa o compromisso para consigo e que a consciência do cuidador acerca da necessidade de se cuidar demonstra que o mesmo precisa estar inserido em um contexto de atenção para a promoção da sua própria saúde.

Neste cenário acadêmico, é necessário ressignificar os conceitos de promoção da saúde, para sensibilizar, o que implica necessariamente em inovar as práticas de ensinoaprendizagem e caracterizar a formação e a atuação dos profissionais da saúde, com vistas a aquisição de habilidades e de atitudes que os façam mais capazes para a vida e para o trabalho, assumindo-se, assim, a educação crítico-reflexiva. ${ }^{2}$ Assim, a sensibilização para a importância do cuidar de si precisa ser estimulada junto aos acadêmicos com vistas à promoção de sua saúde como futuros profissionais de enfermagem.

Para que se possam efetivar estas reflexões, é importante proporcionar aos acadêmicos de enfermagem um ambiente o qual permita a expressão dos mesmos sobre suas expectativas de vida. 0 ambiente universitário faz parte do contexto da vida acadêmica dos futuros profissionais de Enfermagem, no qual vivem, convivem, trocam e adquirem experiências com a natureza e os seus semelhantes, dentro das redes interrelacionais, criando e repadronizando os hábitos de vida. ${ }^{3}$

Vale ressaltar que, no ensino de enfermagem, o processo educativo precisa ser comprometido com o outro, com seus valores e suas relações, pautado na reciprocidade e solidariedade, possibilitando a construção de uma conduta ética, cujo profissional, ao término de sua formação, seja capaz de reconhecer, em cada ação de cuidado, a necessidade e complexidade do ser humano. ${ }^{4}$

Neste sentido o estudo justifica-se como relevante, pois propicia aos acadêmicos de enfermagem momentos de reflexão, encontro e cuidado, de modo a sensibilizar futuros profissionais para as práticas que envolvem o cuidar de si, além de apontar possibilidades para novas maneiras de ensinar na enfermagem.

Sendo assim o referencial utilizado neste estudo desenvolveu conceitos de ser humano - acadêmico de enfermagem; ambiente - ambiente universitário; promoção da saúde; enfermagem/enfermeira; cuidar em enfermagem e cuidar de $\mathrm{si}^{3,5-9}$, pois entende-se que este referencial é adequado para análise dos dados deste estudo.

A questão norteadora que guiou o estudo foi: quais foram às contribuições dos encontros de sensibilização e cuidado relatadas pelos acadêmicos de enfermagem de uma 


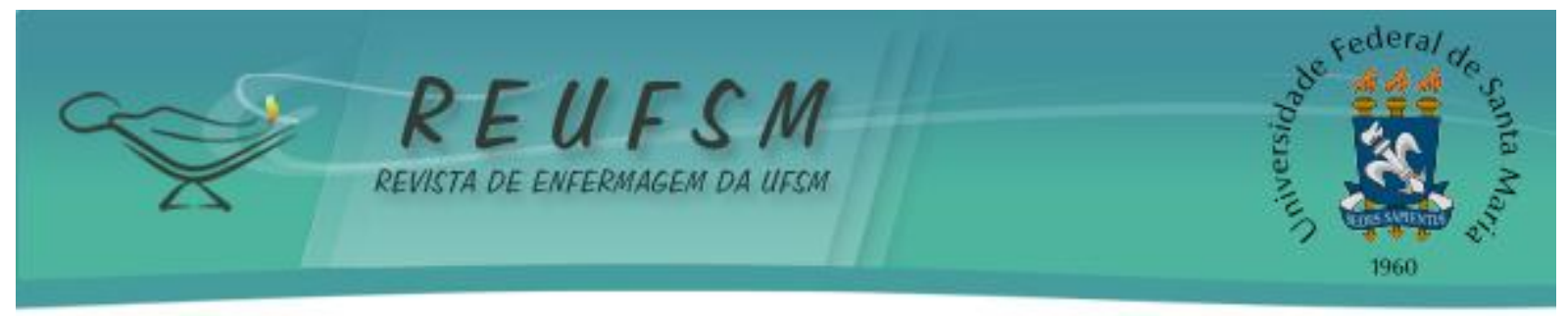

universidade pública do sul do Brasil? E assim, o objetivo deste texto é relatar as contribuições que os encontros de sensibilização e cuidado proporcionaram aos acadêmicos de enfermagem de uma universidade pública do sul do Brasil.

\section{MÉTODO}

Trata-se de uma pesquisa qualitativa, de abordagem Convergente Assistencial (PCA), sendo necessário que a mesma seja desenvolvida no espaço onde ocorrem as relações sociais. É compreendida e realizada em articulação com as ações que envolvem pesquisadores e demais pessoas representativas da situação a ser pesquisada, numa relação de cooperação mútua. ${ }^{10}$ Essa é uma modalidade de pesquisa que, ao mesmo tempo em que se coletam os dados, cuida-se dos participantes. Uma das principais características da PCA consiste na participação dos envolvidos no espaço da pesquisa. Desta forma, a escolha das estratégias utilizadas faz a diferença para a coleta dos dados, evidenciando que esta tem dupla intencionalidade, a de produzir construções científicas nas atividades de pesquisa e a de favorecer o progresso da saúde dos informantes pela prática do cuidado de enfermagem. ${ }^{10}$

Os passos da PCA são: fase de concepção que compreende a introdução da temática, o objetivo do estudo, a revisão de literatura e referencial teórico adotado (citado anteriormente); fase de instrumentação, perscrutação e análise, contemplando nesta fase a organização metodológica da pesquisa; fase de interpretação que indica os resultados, discussão e as considerações finais do estudo.

Assim, seguindo a fase de instrumentação, perscrutação e análise, neste estudo participaram 42 acadêmicos, ou seja, $70 \%$ do total de alunos matriculados na $2^{\text {a }}$ e $3^{\text {a }}$ fases do curso de enfermagem. O critério para inclusão da participação foi que os acadêmicos estivessem devidamente matriculados nas fases referidas.

Todos os acadêmicos, das fases citadas, foram convidados a participar do estudo e, neste momento iniciou-se o processo de aproximação com os mesmos, o qual foi importante e imprescindível para que os mesmos pudessem conhecer a proposta da pesquisa. Os acadêmicos sugeriram os dias e horários para os encontros.

A coleta dos dados deu-se durante quatro encontros não consecutivos, intitulados "Encontros de sensibilização e cuidado", e para tanto, utilizou-se como estratégias de coleta de dados dinâmicas de grupo, confecções de cartazes e atividades lúdicas. As falas dos participantes foram gravadas em aparelho digital Mp4, e posteriormente transcritas em diário de campo.

Em cada encontro foram abordados temas específicos: a importância do cuidar de si; o elo entre o cuidar de si, a promoção da saúde, a prevenção de doenças e evitabilidade do sobrepeso; hábitos saudáveis em seu cotidiano; e validação dos dados coletados.

É oportuno mencionar que o cuidado de enfermagem realizado durante os encontros foram pautados na escuta, na organização do ambiente de forma acolhedora e descontraído, no oferecimento de lanches saudáveis e, principalmente, pela sensibilidade na atenção aos participantes.

A organização dos dados seguiu os critérios da Pesquisa Convergente Assistencial, sendo analisados através do referencial teórico citado anteriormente, e apoiado na literatura pertinente à temática em estudo.

Legalmente, foram respeitadas as determinações da Resolução $n^{\circ}$ 196/96 do Conselho Nacional de Saúde. Assim, a inclusão dos acadêmicos de Enfermagem na pesquisa seguiu as determinações previstas nessa Resolução, que dispõe sobre as diretrizes e normas regulamentares da pesquisa envolvendo seres humanos, especialmente no que se refere ao Termo de Consentimento Livre e Esclarecido (TCLE). Foi garantida aos participantes, por meio do TCLE, a liberdade de participar ou não e de desistir a qualquer momento do estudo. Para preservar o anonimato os participantes foram nomeados com letras do alfabeto. 0 


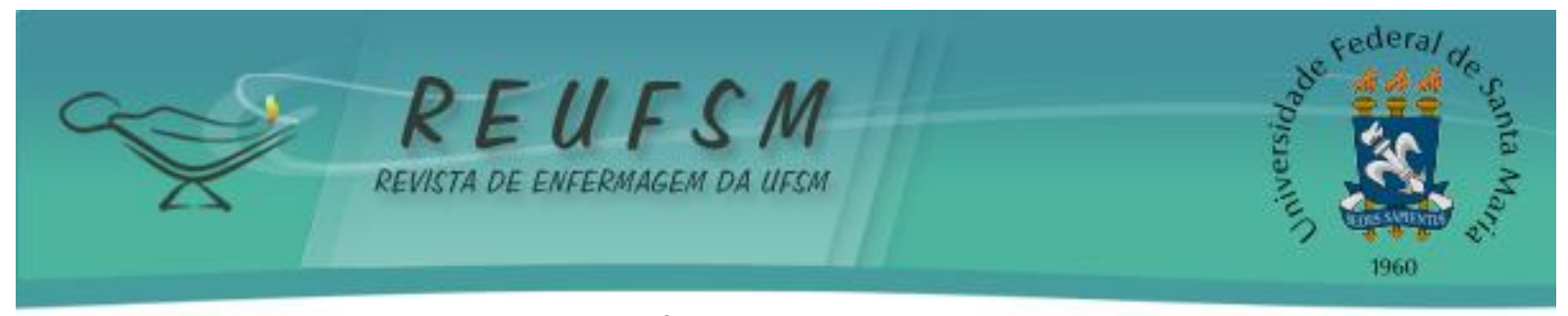

projeto foi avaliado pelo Comitê de Ética em Pesquisa da Universidade Federal de Santa Catarina, sendo aprovado em 30 de maio de 2008 sob o número de protocolo 69/08.

\section{RESULTADOS E DISCUSSÃO}

As discussões nos encontros foram alicerçadas em questões sobre o cuidar de si e a promoção da saúde, e estas permeavam qualquer uma das ações de cuidado, assim como, os aspectos de relacionamento envolvendo o próprio grupo, as situações e as relações entre os participantes. Baseados nos dados construíram-se três temáticas a serem apresentadas: o ambiente e a ludicidade: estratégia para o ensino; a alimentação e autoestima no cotidiano acadêmico; contribuições dos encontros de sensibilização e cuidado.

\section{O ambiente e a ludicidade: estratégia para o ensino}

Antes de o encontro dar início o ambiente da sala onde foram realizadas as atividades era preparado para recepcionar os acadêmicos. 0 movimento de recepção dos acadêmicos na porta da sala fazia com que percebessem o acolhimento. $E$, ao terem esta impressão, observaram que a sala estava decorada com balões no teto, com características de um ambiente diferenciado. Em relação a isto um acadêmico referiu:

\section{É aqui a oficina? Achei que era uma festa [...]. (L) [...] que local agradável e ao mesmo tempo jovem. (A)} comentário:

Da mesma forma ao perceber o ambiente sonoro, outro acadêmico fez o seguinte

Nossa professora! [...] a senhora não vai colocar músicas de relaxamento? Por que estas me fazem dormir [...]. Já percebi que nossa tarde será descontraída. (M)

Observou-se que a interação dos participantes, com o meio ambiente, procura dar condições para favorecer o relaxamento e a descontração. Isso ficou evidenciado quando conseguiram relaxar e se concentrar para realizar as atividades propostas. 0 meio ambiente é o contexto que permeia e afeta a vida do ser humano, o qual envolve condições e influências internas e externas a estes e apresenta uma infinita diversidade, além de proporcionar à pessoa a possibilidade de relacionar-se com outro ser humano, com grupos e com a sociedade como um todo. ${ }^{6}$

Por meio da ludicidade observa-se que a mesma pode proporcionar o bem-estar, sendo elemento qualificador do cuidado de enfermagem que se expressa com criatividade e sensibilidade no ato de cuidar da enfermeira. Destarte, quando concebemos o lúdico no cuidado, pensamos que elementos como a alegria, a descontração, o sorriso, são essenciais no cuidado de enfermagem. ${ }^{11}$

Ao iniciar as atividades dos encontros formaram-se grupos por afinidades, implicando em movimentos diferenciados perante o mesmo desafio, enquanto alguns iniciaram a atividade dialogando sobre o desenvolvimento do mesmo, outros buscavam os materiais disponibilizados como forma de inspiração para expressarem o que pensavam no momento. A partir destas formas diferenciadas de atitude desenvolviam o desafio proposto.

A forma pela qual foram conduzidos os encontros demonstrou que as atividades lúdicas podem favorecer a criatividade, o relaxamento, a troca de conhecimentos e o entrosamento entre os participantes. O lúdico no cuidado configura-se como restaurador 


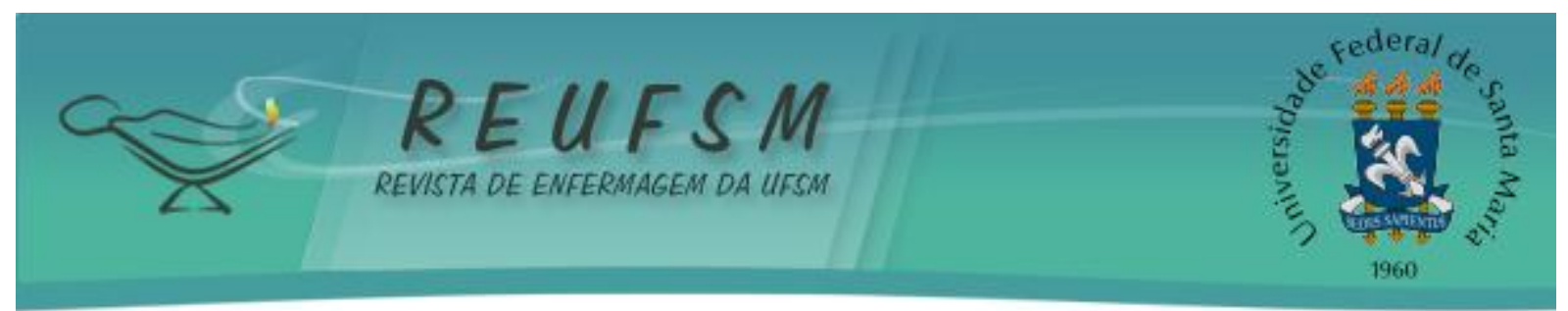

da saúde das pessoas, facilitando a interação, por meio do desenvolvimento intra e interpessoal, promovendo o processo de socialização e comunicação. ${ }^{11}$

No desenvolvimento das dinâmicas, de um modo geral, foi observado que alguns participantes apresentavam mais habilidade para falar e se expressar em público, enquanto outros não; alguns se sentiam incomodados em compartilhar suas percepções. Durante as atividades os acadêmicos tiveram que lançar mão da capacidade criativa, não só com as situações apresentadas, mas também como deveriam utilizar materiais disponíveis. Perceberam que aprender pode ser uma atividade divertida, aonde podem compartilhar momentos de conversas, de risadas, descontração e imaginação. E para isso buscaram em seus conhecimentos prévios e em suas histórias de vida (pessoal e profissional) formas de atuação. "Criar é uma necessidade vital humana, que transforma o mundo, faz o mundo humano, faz o próprio homem, para o que precisamos alcançar a consciência prática". ${ }^{12: 228}$

As estratégias de ensino podem possibilitar e/ou ampliar o desenvolvimento de habilidades interpessoais em estudantes de enfermagem e estas são importantes, pois nem todos trazem as habilidades de se relacionar em sua bagagem pessoal, embora seja imprescindível o desenvolvimento desta na relação enfermeiro/paciente. ${ }^{13} \mathrm{E}$ em encontros os quais utilizam-se estratégias alternativa, como o lúdico, o acadêmico pode expressar-se verdadeiramente e aprender neste contexto como as próprias situações da vida.

\section{A alimentação e auto-estima no cotidiano acadêmico}

Em cada encontro servia-se um lanche com o intuito de descontração, integração entre os participantes, reforçando a importância da alimentação saudável. Além disso, o ato de alimentar-se saudavelmente deve trazer prazer e não ser algo punitivo. Quando se comentava acerca da alimentação saudável alguns acadêmicos se colocavam de maneira a fazer uma auto-avaliação e outros, como se fosse uma forma de justificar perante o grupo suas atitudes cotidianas demonstrando sutilmente que cuidavam de si:

A gente tenta comer bem na maioria das vezes eu e a M. vamos para academia todo santo dia [...] a R. e a C. fazem yoga e a A. trabalha bastante e acaba chegando tarde em casa e fica cansada para isso, mas ela come bastante frutinha [...]. (P)

As meninas no meu grupo, algumas malham [...] e tentamos comer bem hoje, ainda comi sorvete antes de vir para cá [...]. (S)

Um dos questionamentos feitos durante as atividades era em relação ao cuidar de si e se todos tinham clareza sobre este cuidado, e por que se tornava tão difícil no cotidiano? Durante uma das atividades os acadêmicos foram estimulados a realizarem algumas reflexões acerca do cuidar de si, e algumas colocações chamaram a atenção:

Nós achamos importante também cuidar da auto-estima [...] $O$ que é cuidar da auto-estima? É viver bem com a gente mesmo, eu acho [...]. (A)

Compreende-se que, através deste relato, as questões voltadas para a autoimagem, a auto-estima, as formas pelas quais vivem seu cotidiano, algumas potencialidades e fragilidades pessoais interferem diretamente no autocuidado. Neste sentido, ações de autocuidado são importantes, pois constituem em prática de atividades 


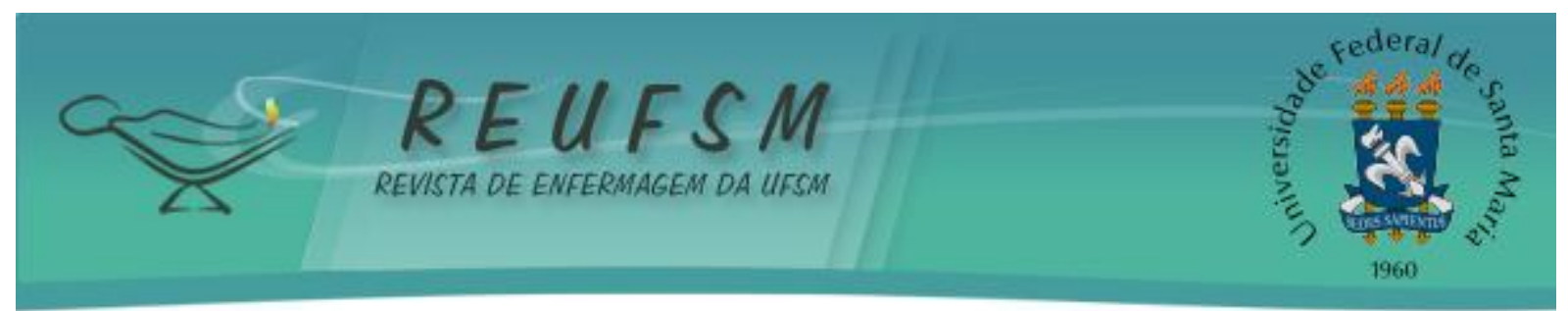

que os indivíduos desempenham de forma definida em seu próprio benefício com o propósito de manter a vida, a saúde e o bem-estar. ${ }^{14}$

Percebeu-se que a participação de todos, nesta temática, foi intensa, pois através dos questionamentos e das reflexões sobre questões gerais do cotidiano colocaram suas realidades. Dessa forma, foi possível identificar que houve um grande interesse e comprometimento dos participantes. Esta reflexão, pautada na dialética, demonstra que a sensibilidade é poder olhar o outro e saber qual a melhor maneira de ensinar e aprender a ser e fazer enfermagem. ${ }^{15}$

0 diálogo junto aos acadêmicos precisa ser sensível à escuta de relatos pessoais que são importantes para o desenvolvimento de um futuro profissional do cuidado, o enfermeiro, criando, desta forma, a possibilidade de reflexão sobre as atitudes cotidianas.

\section{Contribuições dos encontros de sensibilização e cuidado}

Algumas colocações reforçaram o quanto os encontros de sensibilização e cuidado foram importantes para todos os participantes, principalmente porque puderam refletir acerca de seus hábitos e cotidianos acadêmicos em um ambiente acolhedor e lúdico.

Foram momentos de muita descontração, você conseguiu comandar a atividade de maneira muito gostosa. Sempre procurei manter hábitos saudáveis e depois dos encontros continuei com eles e com mais vontade. (M)

Os encontros foram muito agradáveis e auxiliaram-me a refletir sobre o Cuidar de $\mathrm{Si}$, pois como enfermeiros pensamos muito nos outros e esquecemos de nós. $(\mathrm{G})$

$\mathrm{Na}$ validação dos encontros de sensibilização e cuidado compreendeu-se que os acadêmicos ficaram satisfeitos com a interpretação de suas respostas e que se sentiam valorizados por terem participado de um processo de pesquisa, e que suas opiniões e falas foram consideradas importantes.

Esse fato evidenciou que não só os objetivos da pesquisa foram alcançados, mas também, por ter provocado a reflexão sobre seus hábitos e o que fazem para cuidar de si e promover a sua própria saúde. Então, sensibilizar é, também, provocar pequenas mudanças e que podemos começar com o pensar sobre si mesmo. Nesta perspectiva, o cuidar de si pressupõe o exercício da afetividade, uma necessidade humana de trocar e relacionar-se com os outros de forma amistosa, cordial e agradável, para expressão de seus sentimentos. Está diretamente relacionado com a promoção da saúde, tanto física, quanto mental e social, em que a responsabilidade é principalmente do indivíduo, podendo estar amparado por outros indivíduos, profissionais de saúde, sistema de saúde ou da própria natureza. ${ }^{9}$

Através de alguns relatos feitos na fase de validação dos dados da pesquisa, observou-se que nos encontros de sensibilização e cuidado os acadêmicos puderam refletir individualmente sobre seus estilos de vida e como podem fazer a diferença para buscar a promoção da saúde e o cuidar de si.

Estes encontros fizeram com que analisasse o estilo de vida o qual estava levando e percebendo que não adiantava só saber o que é Cuidar de Si sem colocar em prática. (P)

Os encontros realizados fizeram com que analisasse melhor a maneira pela qual cuido de mim. Vi que hoje eles não fazem tanto 


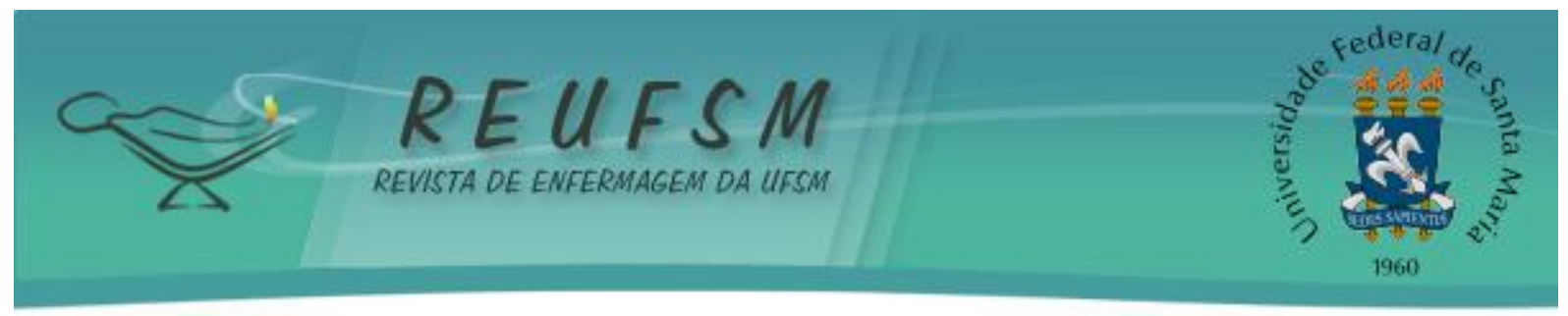

efeito, mas futuramente podem contribuir para o surgimento de algumas doenças. É claro que, principalmente na profissão na qual queremos nos inserir, o Cuidar de Si é necessário para realizar o cuidado com o outro. E, o Cuidar de Si inicia-se com hábitos saudáveis. (M)

Percebeu-se que a dimensão do cuidar e do cuidar de si, envolve um aspecto importante para que este se efetive, que é o diálogo entre os seres humanos envolvidos, sendo evidenciado na prática educativa como uma das maneiras de abrir a possibilidade de novos olhares. "O ato de educar e o ato de cuidar se inter-relacionam por meio de um processo dinâmico e dialógico, o qual se configura como um processo cuidativo". ${ }^{16: 344}$

Neste sentido, o processo educativo significativo pode ser uma das formas de fomentar a sensibilidade para um cuidado humanizado, pois demonstra que o acadêmico sentindo-se valorizado poderá também valorizar o outro, e neste caso, os futuros profissionais de enfermagem poderão ser mais sensíveis no cuidado com o outro. Sendo assim, a adoção de uma filosofia do cuidado humanizado é primordial na formação, pois os acadêmicos têm a oportunidade de familiarizem-se com esta forma de cuidar, e desenvolvendo práticas humanizadoras durante sua vivência acadêmica. ${ }^{17}$

\section{CONSIDERAÇÕES FINAIS}

Provocar mudanças em nossas vidas não é tarefa fácil, porém podem ser provocadas através de sensibilizações. De modo que um dos papéis do enfermeiro é sensibilizar as pessoas para a promoção da saúde e de um viver saudável.

Assim, os encontros de sensibilização e cuidado possibilitaram uma construção visível de cuidado aos acadêmicos de Enfermagem. Proporcionando a identificação de possibilidades e fragilidades em relação ao cuidar de si no cotidiano de vida pessoal e acadêmica.

O despertar para outra dimensão da realidade, diferente do cotidiano acadêmico, impulsionou os participantes ao exercício da reflexão e da crítica, pois durante os encontros procuraram participar ativamente e expor suas ideias e opiniões, demonstrando assim, suas especificidades de ver, estar e perceber o mundo, como um todo, e não somente sua vida universitária.

Assim, através das dinâmicas vivenciadas identificaram-se, nos participantes, as suas crenças e os medos, bem como suas preocupações, e a importância das relações, dos convívios sociais. Por meio de atividades lúdicas, foi possível perceber as observações pessoais e seu modo de viver no meio ambiente, o que revelam fatos que podem ou não contribuir para a saúde ou a doença, além de ser uma das estratégias para o ensino da enfermagem.

Contudo, ficou claro que refletir sobre a realidade, de forma crítica criativa, é a melhor maneira de buscar a expressão do potencial para além da reflexão e a transformação de alguns conceitos do dia a dia do enfermeiro, em relação à promoção da saúde e o cuidar de si.

\section{REFERÊNCIAS}

1. Schossler T, Crossetti MG. Cuidador domiciliar do idoso e o cuidado de si: uma análise através da teoria do cuidado humano de Jean Watson. Texto \& Contexto Enferm. 2008;17(2):280-7.

2. Silva KL, Sena RR, Grillo MJCI, Horta NC, Prado PMC. Educação em enfermagem e os desafios para a promoção de saúde. Rev Bras Enferm. 2009;62(1):86-91. 
3. Sebold LF. Acadêmicos de enfermagem: o cuidar de si para promoção da saúde e evitabilidade do sobrepeso [dissertação]. Florianópolis: UFSC; 2008. 80 p.

4. Carneiro AD, Costa SFG, Pequeno MJP. Disseminação de valores éticos no ensino do cuidar em enfermagem: estudo fenomenológico. Texto \& Contexto Enferm [internet]. 2009 dez [acesso em 2012 jun 4];18(4):722-30. Disponível em: http: //www.scielo.br/pdf/tce/v18n4/14.pdf.

5. Nightingale F. Notas sobre enfermagem: o que é e o que não é. São Paulo: Cortez; 1989.

6. Carraro TE. Resgatando Florence Nightingale: uma trajetória da Enfermagem junto ao ser humano e sua família na prevenção de infecções [dissertação]. Florianópolis: UFSC; 1994.

7. Carraro TE. Desafio secular: mortes maternas por infecções puerperais. Pelotas: UFPel; Florianópolis: UFSC;1999.

8. Radünz V. Cuidando e se cuidando: fortalecendo o "self"do cliente oncológico e o "self"da enfermeira [dissertação]. Florianópolis: UFSC; 1994.

9. Radünz V. Uma filosofia para enfermeiros: o cuidar de si, a convivência com a finitude e a evitabilidade do burnout [tese]. Florianópolis: UFSC; 1999.

10. Trentini M, Paim L. Pesquisa em enfermagem: uma modalidade convergenteassistencial. $2^{\mathrm{a}}$ ed. Florianópolis: UFSC; 2004.

11. Beuter M, Alvim NAT. Expressões lúdicas no cuidado hospitalar. Esc Anna Nery Rev Enferm. 2010;14 (3):567-74.

12. Reibnitz K S, Prado ML. Inovação e educação em enfermagem. Florianópolis: Cidade futura; 2006.

13. Kestenberg CCF, Reis MSA, Motta WC, et al. Cuidando do estudante e ensinando relações de cuidado de enfermagem. Texto \& Contexto Enferm. 2006;15(Esp):193-200.

14. Soares CS, Santos I dos, Berardinelli LMM. Obesity as a social problem: identifying guidance needs of nursing for self-care. Rev Enferm UFPE [internet]. 2010 [acesso em 2011 dez 6];4(1):18-27. Disponível: http://sumarios.org/sites/default/files/pdfs/32148_4138.PDF.

15. Terra MG, Padoin SMM, Gonçalves LHT, Santos EKA, Erdmann AL. O dito e o não-dito do ser-docente-enfermeiro/a na compreensão da sensibilidade. Rev Bras Enferm. 2008;61(5):558-64.

16. Ferraz F, Salum NC, Carraro TE, Radünz V, Espinosa LMM. Educação permanente no trabalho como um processo educativo e cuidativo do sujeito-cuidador. Rev Gaúcha Enferm. 2006;27(3):344-50.

17. Lazzari DD, Jacobs LG, Jung W. Humanização da assistência na enfermagem a partir da formação acadêmica. Rev Enferm UFSM [internet]. 2012 [acesso em 2012 abr 8];2(1):11624.Disponível:http://cascavel.ufsm.br/revistas/ojs2.2.2/index.php/reufsm/article/view/4592/3130.

Data de recebimento: 26/04/2012

Data de aceite: 15/06/2012

Contato com autor responsável: Luciara Fabiane Sebold

Endereço: Rua das Roseiras, 685 Bosque das Mansões. São José - Santa Catarina

CEP: 88108-460

E-mail: fabisebold@gmail.com 